\title{
Hidratación de los cementos de aluminatos de calcio. Parte II: Efecto de las adiciones de silice y alumina
}

\author{
J.M. RIVAS MERCURY ${ }^{a}$, A. H. DE AZA ${ }^{a}, X$. TURRILLAS ${ }^{b}$, P. PENA $^{a}$ \\ aInstituto de Cerámica y Vidrio, CSIC, Cantoblanco. Madrid, España. \\ ${ }^{\mathrm{b}}$ Instituto de Ciencia de la Construcción Eduardo Torroja, CSIC. Madrid España.
}

\begin{abstract}
El presente trabajo es una revisión sobre el proceso de hidratación de los cementos de aluminatos de calcio, de alto contenido en alúmina, con adiciones de microsílice y alumina. En el se describen los principales productos de hidratación de estos materiales. Así mismo se exponen los mecanismos de reacción que han sido establecidos por distintos autores utilizando técnicas diversas. Finalmente se describe el proceso de deshidratación térmica de estos materiales.
\end{abstract}

Palabras clave: cementos de aluminato de calcio, hidratación, microsílice, alumina activada.

Calcium aluminate cements hydration. Part II: Effect of silica and alumina additions.

The present work is a literature review on the hydration process of calcium aluminate cements with high alumina contents with alumina and microsilica additions. The main hydrations products of these materials are described as function of temperature. Their reaction mechanisms, studied through different methods and techniques by several authors are discussed. The products of dehydration at high temperatures are also described.

Key words: calcium aluminate cements, hydration, microsilica, activated alumina

\section{INTRODUCCION}

Actualmente en la formulación de hormigones refractarios se emplean cementos de aluminatos de calcio de elevado contenido en alúmina (70-80\% de $\mathrm{Al}_{2} \mathrm{O}_{3}$ ) como aglomerante hidráulico en la matriz. Esto se debe, a la elevada resistencia mecánica que presentan los hormigones y morteros de estos materiales después de 24 horas. Una forma de mejorar el comportamiento termomecánico de estos materiales, es disminuir el contenido de cemento $(\mathrm{CaO})$ mediante la adición en la matriz de pequeñas cantidades de sustancias químicas inorgánicas de elevada superficie específica que mejoren las propiedades del hormigón. Estas partículas al rellenar los espacios vacíos entre las partículas de los áridos (agregados) disminuyen considerablemente el contenido de agua y cemento necesarios, lo que mejora el fraguado, densidad, porosidad, la reología, así como la resistencia mecánica a altas temperaturas del material y su comportamiento frente a la erosión y corrosión (1-3).

Durante la hidratación estos sólidos, de pequeño tamaño de partícula y elevada superficie específica, absorben parte del agua disponible para las reacciones de hidratación y/o pueden reaccionar con las partículas del cemento refractario variando el mecanismo y la cinética de hidratación de los aluminatos de calcio presentes.

Entre los aditivos empleados se encuentran $\mathrm{Cr}_{2} \mathrm{O}_{3^{\prime}}, \mathrm{ZrO}_{2^{\prime}}$ $\mathrm{TiO}_{2}, \mathrm{SiC}, \mathrm{Al}_{2} \mathrm{O}_{3^{\prime}}$ y $\mathrm{SiO}_{2}$ amorfa, microsilice o humo de sílice, siendo estas dos ultimas las más utilizadas en los últimos años en el diseño de matrices de hormigones refractarios de alto contenido en alúmina.

En la presente revisión, continuación de una previa sobre hidratación de aluminatos de calcio (4), se trataran en detalle la influencia de partículas de alumina y de sílice sobre los procesos de hidratación de los cementos de aluminatos de calcio.

\section{ALÚMINAS ACTIVADAS CALCINADAS.}

Las alúminas reactivas usadas en la formulación de los hormigones refractarios de bajo contenido en cemento, se producen por purificación y calcinación (4-5) de gibbsitas procedentes del proceso Bayer $\left(\mathrm{Al}(\mathrm{OH})_{3}{ }^{*}, \mathrm{AH}_{3}\right)$ y se clasifican según sus contenidos de $\mathrm{Na}_{2} \mathrm{O}$ y niveles de impurezas, en tres categorías:

a) Alúminas calcinadas con contenidos normales de $\mathrm{Na}_{2} \mathrm{O}$ con $0,18-0,55 \%$ en peso de $\mathrm{Na}_{2} \mathrm{O}$ y $99,0-99,5 \%$ en peso de $\mathrm{Al}_{2} \mathrm{O}_{3}$;

b) Alúminas calcinadas de bajo contenido en $\mathrm{Na}_{2} \mathrm{O}(<0,1 \%$ en peso ) $\mathrm{y} \approx 99,7 \%$ en peso de $\mathrm{Al}_{2} \mathrm{O}_{3}$;

c) Alúminas de calcinadas de alta pureza con bajos contenidos de $\mathrm{Na}_{2} \mathrm{O}$ y $99,9 \%$ en peso de $\mathrm{Al}_{2} \mathrm{O}_{3}$.

Estas alúminas de elevada superficie específica, presentan elevada reactividad y sinterizan a temperaturas relativamente bajas, lo que acelera la velocidad de sinterización del hormigón, sin embargo no presentan buenas propiedades hidráulicas. Por esto es necesario, además del control de los contenidos de $\mathrm{Na}_{2} \mathrm{O}$, una buena distribución del tamaño de partículas de forma que garantice su inclusión en los intersticios de la matriz refractaria, mejorando así, el empaquetamiento de partículas y la densidad en verde del refractario. En la Tabla 1, se recogen algunas propiedades físico-químicas de alúminas reactivas comerciales.

* En la química del cemento se suele simplificar la formulación de las fases mineralógicas presentes teniendo en cuenta la siguiente nomenclatura o abreviatura: $\mathrm{S}$

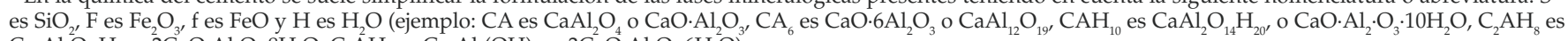
$\mathrm{Ca}_{2} \mathrm{Al}_{2} \mathrm{O}_{13} \mathrm{H}_{16}$ o $2 \mathrm{CaO} \cdot \mathrm{Al}_{2} \mathrm{O}_{3} \cdot 8 \mathrm{H}_{2} \mathrm{O}, \mathrm{C}_{3} \mathrm{AH}_{6}$ es $\mathrm{Ca}_{3} \mathrm{Al}_{2}(\mathrm{OH})_{12}$ o $\left.3 \mathrm{CaO}_{2} \cdot \mathrm{Al}_{2} \mathrm{O}_{3} \cdot 6 \mathrm{H}_{2} \mathrm{O}\right)$. 
TABLA 1 - PROPIEDADES FÍSICO-QUÍMICAS DE ALÚMINAS REACTIVAS COMERCIALES.

\begin{tabular}{|c|c|c|c|c|c|c|}
\hline \multirow{3}{*}{$\begin{array}{c}\text { Análisis } \\
\text { Típicos } \\
\text { Análisis } \\
\text { Químicos } \\
\text { (\% en peso) }\end{array}$} & \multicolumn{6}{|c|}{ Contenidos de $\mathrm{Na}_{2} \mathrm{O}$} \\
\hline & \multicolumn{2}{|c|}{ Bajo } & \multicolumn{2}{|c|}{ Intermedio } & \multicolumn{2}{|c|}{ Normal } \\
\hline & CT-3000 SG & RAC45B** & A- $35^{*}$ & $\mathrm{C}^{2} 4 \mathrm{~B}^{* *}$ & A-2 & $\begin{array}{l}\text { C- } \\
70^{* *}\end{array}$ \\
\hline $\mathrm{Al}_{2} \mathrm{O}_{3}$ & 99,8 & 99,8 & 99,6 & 99,8 & 99,6 & 99,5 \\
\hline $\mathrm{Na}_{2} \mathrm{O}$ & 0,08 & 0,04 & $0,20 / 0,28$ & 0,18 & 0,28 & 0,40 \\
\hline $\mathrm{SiO}_{2}$ & 0,03 & 0,01 & 0,01 & 0,02 & 0,01 & 0,03 \\
\hline $\mathrm{Fe}_{2} \mathrm{O}_{3}$ & 0,02 & 0,01 & 0,01 & 0,01 & 0,01 & 0,03 \\
\hline $\mathrm{D}_{50}(\mu \mathrm{m})$ & 0,8 & 0,2 & 4,8 & 0,5 & 0,5 & 2,3 \\
\hline $\begin{array}{l}\text { Se BET ( } \\
\left.\mathrm{m}^{2} / \mathrm{g}\right)\end{array}$ & 7,5 & 8,0 & 1,5 & 7,0 & 0,8 & 0,6 \\
\hline
\end{tabular}

$\left({ }^{*}\right)$ - Aluminas reactivas de AlCOA - (Aluminium company of America) $\left.{ }^{* *}\right)$ - Aluminas reactivas de ALCAN - (Aluminium of Canada )

2.1. Efecto de la adición de alúminas calcinadas reactivas sobre la hidratación de los aluminatos de calcio.

Las características físico-químicas de las alúminas reactivas $\left(\alpha-\mathrm{Al}_{2} \mathrm{O}_{3}\right)$ juegan un papel importante en las propiedades hidráulicas, reológicas y de alta temperatura de los cementos refractarios (6-7). Por esta razón, sus efectos sobre el comportamiento durante la hidratación de aluminatos de calcio y de sus mezclas a diferentes temperaturas $\left(\mathrm{CaAl}_{2} \mathrm{O}_{4}-\mathbf{C A}, \mathrm{CaAl}_{4} \mathrm{O}_{7}-\mathbf{C A}_{2}\right)$ ha sido objeto de estudio por algunos autores.

Rettel y col.(8), basandose en los estudios realizados por Bushnell y Sharp (9-11) sobre el fraguado anormal de los cementos refractarios de aluminatos de calcio a bajas temperaturas, investigaron la hidratación de CA con adiciones de alúmina reactiva ( $80 \%$ en peso) a temperaturas de $15,20,22$ y $30{ }^{\circ} \mathrm{C}$ y relaciones agua $/$ cemento $^{\mathrm{d}}(\mathbf{a} / \mathbf{c})=0,5$, empleando, Difracción de rayos X (DRX) y Calorimetría, concluyeron, que la adición de alúmina reactiva al CA aceleraba fuertemente el proceso de hidratación de este compuesto en todo el rango de temperatura estudiado y que el grado de hidratación de las pastas de $\mathrm{CA}+\mathrm{A}$, era mayor que las del CA puro, además ninguna de las mezclas hidratadas presentó un fraguado anormal durante el estudio. Las fase identificadas por DRX, fueron pequeñas cantidades de $\mathrm{CAH}_{10}$ y $\mathrm{AH}_{3}$ y $_{2} \mathrm{AH}_{8}$ como fase principal a 20 y $25^{\circ} \mathrm{C}$.

Möhmel, Geßner y colaboradores (12-13), investigaron la influencia de la superficie específica y del contenido de $\mathrm{Na}_{2} \mathrm{O}$ de alúminas reactivas, en la hidratación del CA, a las temperaturas de 20 y $50{ }^{\circ} \mathrm{C}$ con relación de a/c de 0,5 empleando Difracción de Rayos X (DRX), Espectrometría de Emisión por Plasma (ICP-AES), Isotermas de Absorción de $\mathrm{N}_{2}$, Análisis Térmico Diferencial (ATD-TG), Calorimetría y Resonancia Magnética Nuclear de Sólidos (RMNS) en atmósfera libre de $\mathrm{CO}_{2}$. Los estudios realizados a $20^{\circ} \mathrm{C}$ mostraron que, las alúminas con elevada superficie específica disminuían el período de inducción, acelerando la cinética de hidratación de las mezclas. También se estableció en estos estudios que, el contenido de $\mathrm{Na}_{2} \mathrm{O}$ influye en la cristalinidad de los hidratos formados, en su composición, en la formación de la capa hidratada superficial de las partículas de aluminato monocálcico $\left(\mathrm{CAH}_{10}\right)$ y en la cantidad de hidróxido de aluminio $\left(\mathrm{AH}_{3}\right)$ cristalino formado. Otro resultado importante de las investigaciones realizadas por estos autores que vale la pena destacar, es la ausencia de una correlación directa entre los contenidos de $\mathrm{Na}_{2} \mathrm{O}$ presentes en las alúminas reactivas empleadas y los productos de hidratación, puesto que, alúminas con características similares, conducían a productos de hidratación y microestructuras diferentes, lo pone de manifiesto la especial atención que se debe tener en cuenta a la hora de seleccionar alúminas calcinadas reactivas para su adición (utilización como aditivo) en los cementos refractarios de aluminatos de calcio, ya que, pequeños cambios en las características físico-químicas de estos materiales pueden afectar a parámetros como el tiempo de fraguado y la resistencia mecánica. Los mismos autores al estudiar el efecto del aumento de la temperatura $\left(50{ }^{\circ} \mathrm{C}\right)$ observaron un aumento de la velocidad de hidratación y de la superficie específica de las pastas estudiadas, el cual lo asociaron a la transformación del $\mathrm{C}_{2} \mathrm{AH}_{8}$ (hexagonal) en $\mathrm{C}_{3} \mathrm{AH}_{6}$ (cúbico) (14-15). Estos mismos autores, estudiaron el efecto de adiciones de alúminas de diferente superficie específica en el comportamiento durante la hidratación de mezclas de CA y $\mathrm{CA}_{2}$, usando las mismas técnicas y métodos descritos anteriormente (16), concluyendo que las adiciones de alúmina aumentan el grado y la velocidad de hidratación del CA y del $\mathrm{CA}_{2}$, independientemente de la cantidad de $\mathrm{CA}_{2}$ adicionado.

Mathieu y col.(17), estudiaron seis tipos de alúminas reactivas con contenidos variables de $\mathrm{Na}_{2} \mathrm{O}$ y diferentes superficies específicas y su relación con la hidratación de un cemento de aluminato de calcio por Calorimetría, (DRX), Microscopia Electrónica de Barrido (MEB) y Análisis Químico de Soluciones. Concluyeron que las alúminas reactivas actúan como aceleradores de la reacción de hidratación del CA, diminuyendo el tiempo del periodo inducción, el tiempo de fraguado y de formación de hidratos, en función de la composición y la superficie especifica de la alúmina reactiva empleada.

Por lo tanto, se puede concluir de estos estudios, que la adición de alúminas reactivas calcinadas con elevadas superficies específicas, a mezclas de CA y $\mathrm{CA}+\mathrm{CA}_{2}$, aceleran la velocidad y el grado de hidratación de los aluminatos de calcio y de sus mezclas presentes en el cemento, requiriendo especial atención el contenido de $\mathrm{Na}_{2} \mathrm{O}$ presente en su composición.

\section{EFECTO DE LAS ADICIONES DE SILICE SOBRE LA HIDRATACIÓN DE LOS ALUMINATOS CALCICOS}

\subsection{El sistema cuaternario $\mathrm{CaO}-\mathrm{Al}_{2} \mathrm{O}_{3}-\mathrm{SiO}_{2}-\mathrm{H}_{2} \mathrm{O}$}

Las relaciones de equilibrio de fases del sistema $\mathrm{CaO}-$ $\mathrm{Al}_{2} \mathrm{O}_{3}-\mathrm{SiO}_{2}-\mathrm{H}_{2} \mathrm{O}$ (C-A-S-H), en condiciones hidrotermales a elevadas temperaturas y presiones, han sido objeto de muchos estudios debido a su gran importancia en geología y petrología (18-19). Sin embargo, son casi inexistentes estudios de las relaciones de equilibrio de fases de este sistema cuaternario a bajas temperaturas y presiones, los cuales sin duda, serian de gran ayuda en la comprensión de la físico-química de las fases que se forman durante el proceso de hidratación y deshidratación en sistemas de cementos de aluminatos de calcio de alto contenido en alúmina con adiciones de sílice coloidal.

Durante la hidratación de un cemento (mezcla de aluminatos cálcicos) con sílice coloidal pueden formarse una serie de fases hidratadas binarias y ternarias dentro de los subsistemas $\mathrm{CaO}-\mathrm{SiO}_{2}-\mathrm{H}_{2} \mathrm{O}(\mathrm{C}-\mathrm{S}-\mathrm{H})$ y $\mathrm{CaO}-\mathrm{Al}_{2} \mathrm{O}_{3}-\mathrm{H}_{2} \mathrm{O}(\mathrm{C}-\mathrm{A}-\mathrm{H})$, así

${ }^{\mathrm{d}}$ Agua / cemento se refiere a la relación agua / producto hidráulicamente activo 
como, compuestos hidratados cuaternarios dentro del sistema $\mathrm{CaO}-\mathrm{Al}_{2} \mathrm{O}_{3}-\mathrm{SiO}_{2}-\mathrm{H}_{2} \mathrm{O}$, que presentan diferentes rangos de estabilidad en función de condiciones experimentales como: temperatura, presión, relación de a $/ \mathrm{c}=($ cemento + sílice $)$ y $\mathrm{pH}$. Experimentalmente se ha verificado la formación de una serie de compuestos denominados hidrogrosularias o hidrogranates de formula general $\mathrm{Ca}_{3} A l_{2}\left(\mathrm{SiO}_{4}\right)_{3-x}(\mathrm{OH})_{4 x}-\left(C_{3} A(S)_{3-X}(H)_{2 X}\right)$ $(0 \leq x \leq 3)$ cuyos extremos son el hidrogranate sin sílice $\left(\mathbf{C}_{3} \mathbf{A H}_{6}\right)$ y la grosularia $\mathrm{Ca}_{3} \mathrm{Al}_{2} \mathrm{Si}_{3} \mathrm{O}_{12}\left(\mathbf{C}_{3} \mathrm{AS}_{3}\right)$. Esta familia de soluciones sólidas se forma por substituciones del tipo $4(\mathrm{OH})^{-} \Leftrightarrow\left(\mathrm{SiO}_{4}\right)^{4-}$ en la estructura de los compuestos finales, siendo $3-\mathrm{x}$ la cantidad de sílice substituida por grupos $(\mathrm{OH})^{-}$en la estructura. Estos compuestos de estructura cúbica, pertenecen al grupo espacial Ia3d (230), en sus estructuras cristalinas los cationes de calcio presentan coordinación dodecaédrica (VIII), los aluminios octaédrica (VI) y los silicios coordinación tetraédrica (IV). La figura 1 muestra los diferentes tipos de coordinación de los cationes calcio, aluminio y silicio en la estructura cristalina del $\mathrm{C}_{3} \mathrm{AH}_{6}$ (a) y del $\mathrm{C}_{3} \mathrm{AS}_{3}(\mathrm{~b})$.

Damidot y Glasser (22) usando métodos de cálculo termodinámicos y estudios realizados anteriormente por Jappy y Glasser (23) sobre la síntesis y la estabilidad de las soluciones sólidas de sílice en la estructura de los hidrogranates, establecieron las relaciones de equilibrio de fases en el sistema $\mathrm{CaO}-$ $\mathrm{Al}_{2} \mathrm{O}_{3}-\mathrm{SiO}_{2}-\mathrm{H}_{2} \mathrm{O}$ a $25^{\circ} \mathrm{C}$ (Figura 2). Estos autores, concluyeron que en este sistema existen 10 puntos invariantes, 18 líneas eutécticas ternarias y 9 hidratos estables. Según estos estudios, dependiendo de la cantidad de sílice que reemplaza a los grupos hidroxilos, en las pastas de cementos hidratadas con adiciones de sílice, pueden formase dos familias de soluciones sólidas denominadas katoita $\mathbf{C}_{3} \mathbf{A}(\mathbf{S})_{3-x}(\mathbf{H})_{2 x}$ (donde $x$ varia entre 1,5 y 3 , se trata de hidrogranates de bajo contenido en sílice, HG-Si-B) y hibschita $\mathbf{C}_{3} \mathbf{A}(\mathbf{S})_{3-\mathrm{x}}(\mathbf{H})_{2 \mathrm{x}}$ (donde $\mathrm{x}$ varia entre 0,2 y 1,5, hidrogranate de alto contenido en sílice HG-Si-A) (24). Además de estas soluciones sólidas se han identificado otros compuestos como la gehlenita hidratada o strätlingite $\left(\mathrm{Ca}_{2} \mathrm{Al}_{2} \mathrm{SiO}_{15} \mathrm{H}_{16}-\mathrm{C}_{2} \mathrm{ASH}_{8}\right)$, la bicchulita $\left(\mathrm{Ca}_{2} \mathrm{Al}_{2} \mathrm{SiO}_{7} \mathrm{H}_{2}-\mathrm{C}_{2} \mathrm{ASH}\right)$, hidróxidos de aluminio cristalinos (gibbsita), geles de hidróxidos de aluminio amorfos, geles de silicatos cálcicos y aluminatos de calcio hidratados.

Un análisis del diagrama de fases propuesto por estos investigadores permite observar, que la fase $\mathrm{C}_{2} \mathrm{ASH}_{8}$ no es estable en presencia de $\mathrm{Ca}(\mathrm{OH})_{2}$, pero si lo es en presencia de $\mathrm{AH}_{3}$ y HG-Si-B y geles de C-S-H, fases detectadas como productos de hidratación de los cementos de aluminatos de calcio. El mismo diagrama muestra que el $\mathrm{C}_{2} \mathrm{ASH}_{8}$ es incompatible con el hidrogranate sin sílice $\left(\mathrm{C}_{3} \mathrm{AH}_{6}\right)$, sin embargo, esta fase puede coexistir en equilibrio con el hidrogranate HG-Si-B y HG-Si-A, geles de C-H-S y una fase acuosa.

Damidot y Glasser teniendo en cuenta el diagrama de fases propuesto, explicaron la discrepancia en los resultados obtenidos por otros investigadores en función de pequeñas variaciones en la composición que pueden llevar a cambios drásticos en la constitución de las fases en equilibrio (ver Figura 2).

3.2.- Efecto de las adiciones de humo de sílice en la hidratación de los aluminatos de calcio.

La sílice coloidal, humo de sílice, o microsilice, es un producto amorfo obtenido por la condensación, filtración y clasificación de los humos del proceso de producción del silicio metálico o del ferro-silicio en hornos de arco eléctrico (25). Este material está formado por partículas esféricas con un tamaño

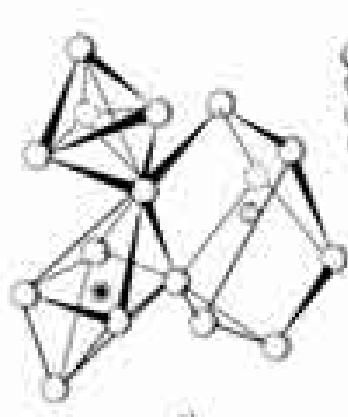

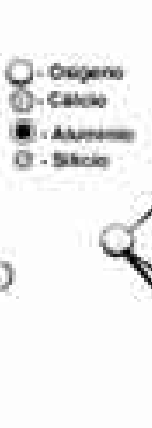

a)

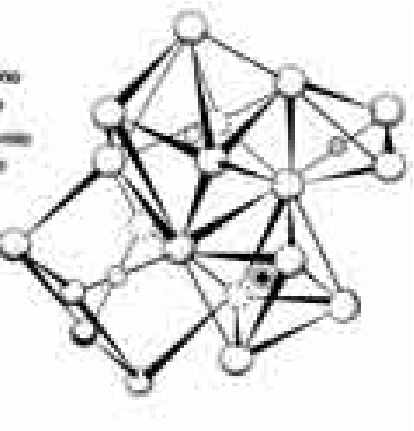

b)
Figura 1 - Estructura cristalina: a) $\mathrm{C}_{3} \mathrm{AH}_{6}(20)$ y b) del $\mathrm{C}_{3} \mathrm{AS}_{3}(21)$, mostrando los poliedros de coordinación de los diferentes cationes, $\mathrm{Ca}^{[\mathrm{VIII}]}$, $\mathrm{Al}^{[\mathrm{VI}]}, \mathrm{Si}^{[\mathrm{IV}]}$.

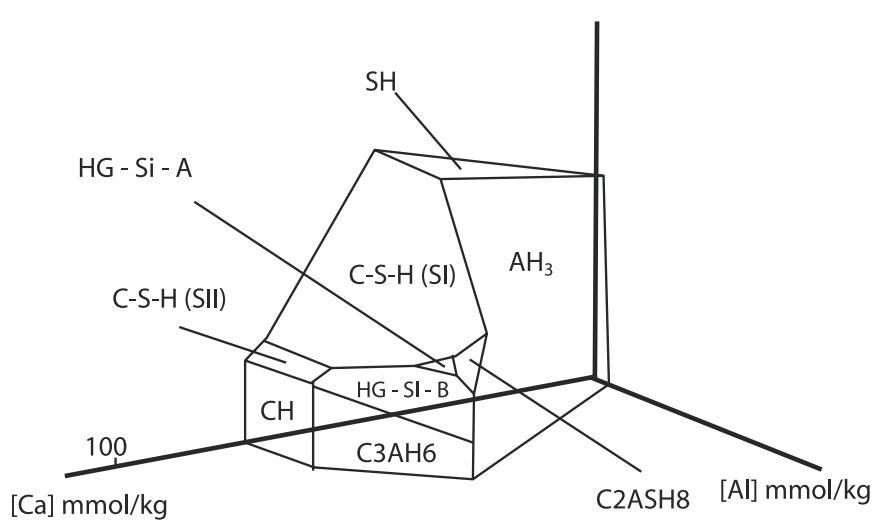

Figura 2 - Diagrama de fases $\mathrm{CaO}-\mathrm{Al}_{2} \mathrm{O}_{3}-\mathrm{SiO}_{2}-\mathrm{H}_{2} \mathrm{O}$ a $25^{\circ} \mathrm{C}$, propuesto por Damidot y Glasser (9). (HG-Si-A y HG-Si-B, denotan hidrogranate de alto contenido en sílice y de bajo contenido en sílice respectivamente).

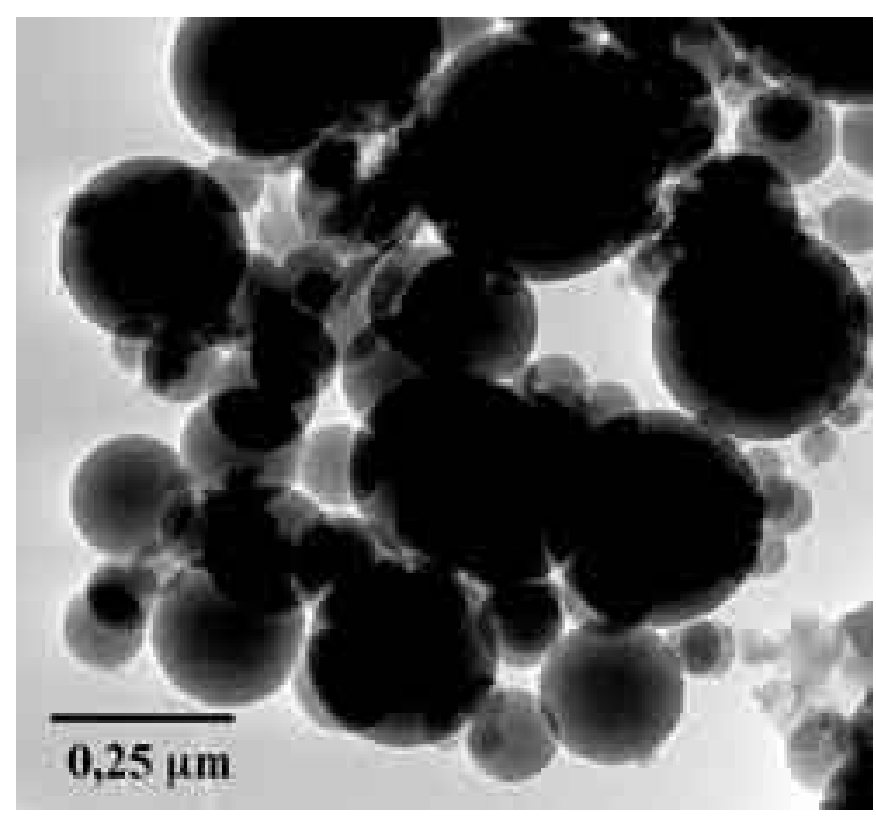

Figura 3 - Micrografia obtenida por microscopia electronica de transmisión de las partículas esféricas de microsilice.

medio del orden de 0,15 micras, y una amplia distribución de tamaño de partícula submicrométricas, y con superficies específicas del orden de $20 \mathrm{~m}^{2} / \mathrm{g}$; sus niveles de pureza varían entre 98.3-93,0 \% en peso de sílice. La Figura 3 muestra la morfología típica de este material. 
Por sus propiedades superficiales, físicas y químicas, la sílice coloidal viene siendo utilizada junto con los cementos de aluminato de calcio como aglomerante hidráulico en la fracción fina de hormigones refractarios. Las adiciones controladas de microsilice a los hormigones refractarios de bajo contenido en cemento, mejoran su fluidez y el factor de empaquetamiento del hormigón, ya que la microsílice tiende a acomodarse entre los intersticios existentes entre los áridos (agregados refractarios de tamaños medios y gruesos) y la fracción mas fina o matriz del hormigón, mejorando la reología del sistema y propiedades tales como la porosidad y la resistencia mecánica en verde del material (26). Sin embargo, una de las limitaciones de su uso en hormigones de bajo contenido en cemento se debe a su tendencia a reaccionar con el $\mathrm{CaO}$ y el $\mathrm{Al}_{2} \mathrm{O}_{3}$ del cemento de aluminatos de calcio a temperaturas superiores a los 1.000 ${ }^{\circ} \mathrm{C}$, para formar anortita $\mathrm{CAS}_{2}$ y gehlenita $\mathrm{C}_{2} \mathrm{AS}$, fases que presentan un punto invariante eutéctico a $\sim 1.380{ }^{\circ} \mathrm{C}$, dentro del sistema ternario $\mathrm{CaO}-\mathrm{Al}_{2} \mathrm{O}_{3}-\mathrm{SiO}_{2}$, lo que limita la aplicación del material a altas temperaturas (27).

La microsílice actúa como agente retardador de la hidratación de los aluminatos de calcio, reteniendo iones de $\mathrm{Ca}^{2+} \mathrm{y}$ $\mathrm{Al}^{3+}$, al formar fases minerales dentro del sistema $\mathrm{CaO}-\mathrm{Al}_{2} \mathrm{O}_{3}-$ $\mathrm{SiO}_{2}-\mathrm{H}_{2} \mathrm{O}$ con estructuras de tipo pseudo-zeolitas (28) o zeolitas (29), $\mathrm{C}_{2} \mathrm{ASH}_{8}$ e hidratos de $\mathrm{CaO}-\mathrm{SiO}_{2}-\mathrm{H}_{2} \mathrm{O}$, a las cuales se atribuye la elevada resistencia mecánica en verde observada en los hormigones refractarios con bajo contenido en cemento. La presencia de estas fases impide que las moléculas de agua químicamente enlazadas sean liberadas abruptamente durante el calentamiento del hormigón en un estrecho intervalo de temperatura, lo que evita el desconchado por explosión (30-31).

Monsen y colaboradores (28), estudiaron mediante DRX, el efecto de adiciones de microsílice sobre la hidratación de pastas de cemento refractario con relaciones de a $/ \mathrm{c}=0,5$ a $40^{\circ}$ $\mathrm{C}$ y $65^{\circ} \mathrm{C}$. Estos autores mostraron que a $40^{\circ} \mathrm{C}$ los productos de hidratación formados en el intervalo de tiempo de 15-20 $h$ estaban constituidos por las fases transitorias $\alpha-C_{2} \mathrm{AH}_{8} \mathrm{y}$ $\beta-\mathrm{C}_{2} \mathrm{AH}_{8}$ que al transcurrir el tiempo se transformaron en gehlenita hidratada $\left(\mathrm{C}_{2} \mathrm{ASH}_{8}\right)$ de baja cristalinidad, siendo esta última fase el principal producto de hidratación después de 3 días de curado. A $65^{\circ} \mathrm{C}$ el proceso de hidratación se acelera formando las mismas fases que en el caso anterior $\left(40^{\circ} \mathrm{C}\right)$, $\mathrm{C}_{3} \mathrm{AH}_{6}$ y $_{2} \mathrm{ASH}_{8}$ como fase mayoritaria. También observaron, que el hidrato $\mathrm{C}_{2} \mathrm{ASH}_{8}$ era estable a la temperatura de $65^{\circ} \mathrm{C}$ y no sufría conversión después de su formación, contradiciendo las afirmaciones de la literatura sobre la inestabilidad de esta fase a temperaturas superiores a $\operatorname{los} 50{ }^{\circ} \mathrm{C}$, atribuyeron este comportamiento a la baja concentración de $\mathrm{Ca}(\mathrm{OH})_{2}$ presente en los cementos de aluminatos de calcio para aplicaciones refractarias (32). El estudio por DRX mostró, el efecto retardador de la microsilice en el proceso de hidratación de las pastas principalmente a $65^{\circ} \mathrm{C}$, al ser comparado con pastas sin microsílice.

Bentsen y col.(33), estudiaron el efecto de la temperatura y el tiempo de hidratación en pastas de cemento ${ }^{7}$, con una relación a/c $=0,5$ y con contenidos de 30 y 50\% de microsilice en el intervalo de temperaturas de $5-100^{\circ} \mathrm{C}$. Los resultados obtenidos están de acuerdo con los estudios realizados por Monsen $^{28}$. La identificación de las fases por DRX mostró, la presencia de hidrato de gelhenita en las pastas endurecidas como fase mayoritaria después de $24 \mathrm{~h}$ de curado en el rango de temperaturas de $40-70{ }^{\circ} \mathrm{C}$. Después de 1 semana de hidratación a temperaturas entre $20-30{ }^{\circ} \mathrm{C}$, se observaron la presencia de $\mathrm{C}_{2} \mathrm{ASH}_{8^{\prime}}$ así como microsílice sin reaccionar. Para explicar la presencia de esta fase $\left(\mathrm{C}_{2} \mathrm{ASH}_{8}\right)$ propusieron la reacción:

$$
\beta-\mathrm{C}_{2} \mathrm{AH}_{8}+\mathrm{S} \cdot \mathrm{nH} \rightarrow \mathrm{C}_{2} \mathrm{ASH}_{8}+\mathrm{n} \mathrm{H}_{2} \mathrm{O}
$$

Otro aspecto importante del trabajo realizado por estos autores es la observación de cambios de intensidad, y dobles picos solapados en las posiciones de $30^{\circ}$ y $10^{\circ}(2 \theta)$ para el $\mathrm{C}_{3} \mathrm{AH}_{6}$, lo que sugiere la formación de soluciones sólidas entre el hidrogranate y la microsílice lo que se puede explicar mediante reacciones como la siguiente aunque estos autores no las formulan de modo explícito.

$$
\mathrm{C}_{3} \mathrm{AH}_{6}+(3-\mathrm{x}) \mathrm{S} \cdot \mathrm{nH} \rightarrow \mathrm{C}_{3} \mathrm{~A}(\mathrm{~S})_{3-\mathrm{x}}(\mathrm{H})_{2 \mathrm{x}} \quad(0 \leq \mathrm{x} \leq 3)
$$

La influencia del $\mathrm{Na}_{2} \mathrm{O}$ presente en la microsilice y su relación con el mecanismo de formación de strätlingita en cementos de alto contenido en alúmina ha sido también objeto de estudio por Ding y colaboradores (34-35) en estas investigaciones se muestra que, a pH elevados ( 12), los iones alcalinos actúan como catalizadores que activan la superficie de la sílice coloidal para formar aniones de silicatos que reaccionan con el cemento hidratado para producir $\mathrm{C}_{2} \mathrm{ASH}_{8}$ (36-37).

Se han realizado otros trabajos de investigación con la finalidad de entender el efecto de las adiciones de sílice y sus implicaciones en la hidratación de los cementos de aluminatos de calcio (38), revelando que el mecanismo de reacción de estos sistemas es más complejo de lo que se pensaba. Se ha observado que el tipo de microsilice empleada, su concentración, contenido de impurezas, tipo de aluminato de calcio y las condiciones experimentales como temperatura y relación agua/cemento empleada, pueden afectar la formación de algunas fases. Así, los productos de reacción en ausencia de $\mathrm{C}_{2} \mathrm{ASH}_{8}$ serian, $\mathrm{C}_{2} \mathrm{AH}_{8}$ que se convierte en $\mathrm{C}_{3} \mathrm{AH}_{6}$, mezclado con soluciones sólidas de tipo $\mathrm{C}_{3} \mathrm{~A}(\mathrm{~S})_{3-\mathrm{x}}(\mathrm{H})_{2 \times}$ y geles del tipo Al-Si-O y sílice sin reaccionar(39-41).

\subsection{Estudio microestructural}

En la bibliografía existen muy pocos estudios sobre la microestructura de los hidratos formados en mezclas de CA con sílice coloidal.

Uno de los mas representativos se debe a Fumo (42) quien en su estudio de la influencia de diferentes adiciones de sílice coloidal sobre la hidratación de CA, utilizando un CA obtenido por el método de combustión con urea, encuentra que en mezclas de (3:1) de CA/Sílice coloidal, con una relación agua/ cemento de 2, la formación de hidratos depende fuertemente de la temperatura, así después de 24 horas a $25^{\circ} \mathrm{C}$ la microestructura del material, Figura $4 a$, presenta dos tipos de cristales: placas hexagonales de gran tamaño que deben corresponder a $\mathrm{C}_{2} \mathrm{AH}_{8}$ y aglomerados de partículas esféricas de pequeño tamaño $\approx 1 \mu \mathrm{m}$ que deben corresponder al $\mathrm{AH}_{3} \mathrm{y}$ a geles de sílice. La misma mezcla después de 10 horas de hidratación a $50{ }^{\circ} \mathrm{C}$ y con la misma relación agua/cemento presenta una microestructura totalmente diferente (Figura $4 \mathrm{~b}$ ), con la formación de cristales con simetría cúbica correspondientes a fases de tipo hidrogranate junto con la formación de aglomerados de partículas esféricas similares a los detectados en la muestra hidratada a $25^{\circ} \mathrm{C}$. 

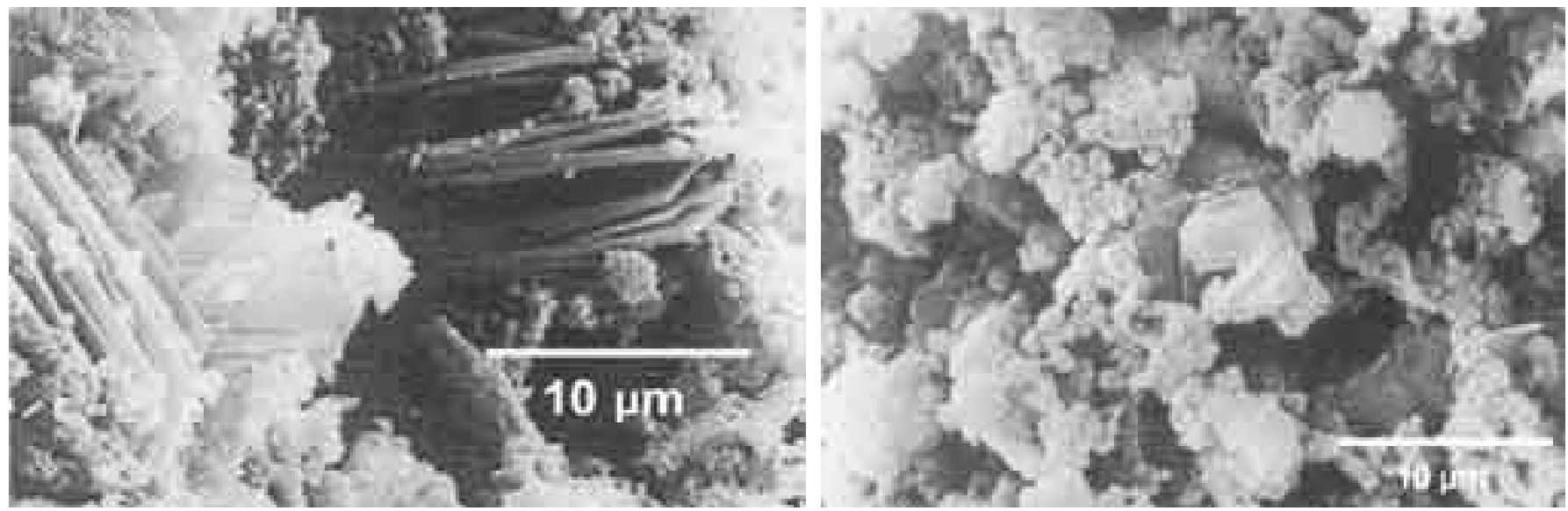

Figura 4 - Hidratación de mezclas (3:1) de CA/Sílice coloidal: (a) - superficie de fractura de la mezcla hidratada durante 24 h a $25^{\circ} \mathrm{C}$ con una relación agua/cemento $=2$. b) - Superficie de fractura de la misma mezcla hidratada durante 10 h a $50{ }^{\circ} \mathrm{C}$ con una relación a/c $=2(42)$.

\section{DESHIDRATACIÓN DE LOS ALUMINATOS CÁLCICOS HIDRATADOS}

Los principales productos de hidratación a temperatura ambiente y tiempos cortos de hidratación de los cementos de aluminatos de calcio son $\mathrm{CAH}_{10} \mathrm{y} \mathrm{AH}_{3}$ amorfo. La temperatura acelera el proceso de conversión de los hidratos hexagonales metaestables $\left(\mathrm{CAH}_{10}, \mathrm{C}_{2} \mathrm{AH}_{8}\right)$ a los hidratos cúbicos estables $\left(\mathrm{C}_{3} \mathrm{AH}_{6}\right)$. Este proceso tiene lugar con cambios de densidad que generan en el material un aumento de la porosidad y variaciones dimensionales que pueden alcanzar el $53 \%$ en volumen y perdidas significativas de resistencia mecánica (43).

La Figura 5a y b muestra la curva de deshidratación típica obtenida por ATD-TG de un cemento de monoaluminato de calcio hidratado $(45,56)$. En la Figura 6 se presenta la proyección bidimensional de evolución mineralógica con la temperatura de la misma muestra obtenida in situ mediante difracción de neutrones (45).

Existen discrepancias entre los resultados obtenidos por los diferentes autores sobre la evolución mineralógica con la temperatura de los diferentes hidratos aunque algunas de las reacciones parece que están bien establecidas:

Durante el calentamiento el $\mathrm{CAH}_{10}$ se deshidrata parcialmente perdiendo agua de cristalización y formando compuestos intermedios metaestables como: $\mathrm{CaO} \cdot \mathrm{Al}_{2} \mathrm{O}_{3} \cdot \mathrm{xH}_{2} \mathrm{O}(\mathrm{x}<10)$ y $\mathrm{C}_{2} \mathrm{AH}_{8^{\prime}}$ los cuales a su vez se descomponen al aumentar la temperatura (o con el tiempo) formando, entre $170-195^{\circ} \mathrm{C}$, la fase cúbica estable $\mathrm{C}_{3} \mathrm{AH}_{6}$. A temperaturas superiores a $195^{\circ}$ $\mathrm{C}$ la pasta de cemento hidratado esta constituida por $\mathrm{C}_{3} \mathrm{AH}_{6} \mathrm{e}$ hidróxido de aluminio (gibbsita - $\mathrm{AH}_{3}$ ).

La gibbsita deshidrata endotérmicamente en el intervalo de temperatura $210-330^{\circ} \mathrm{C}$ formando una fase de muy baja cristalinidad tipo boehmita (AlO.OH) (Fig. 5); en el intervalo $530-560^{\circ} \mathrm{C}$ se observa una nueva reacción endotérmica que se atribuye a la formación de alúminas de transición también con un muy pequeño nivel de cristalinidad (44), ninguna de estas fases transitorias ha sido detectada por DRX o por DN (45) (Fig. 6), las alúminas de transición originan $\alpha$-alúmina en el intervalo de temperaturas entre $1000-1200{ }^{\circ} \mathrm{C}(46,47)$.

$\mathrm{El} \mathrm{C}_{3} \mathrm{AH}_{6}$ presenta un efecto endotérmico en el rango de temperatura de $300-360{ }^{\circ} \mathrm{C}$ (Fig. 5), que se atribuye a la liberación de 4,5 moléculas de agua con la formación de $\mathrm{C}_{3} \mathrm{AH}_{1.5}$ como compuesto intermedio metaestable $(47,48)$. Otros autores sugieren que el $\mathrm{C}_{3} \mathrm{AH}_{6}$ se descompone en $\mathrm{C}_{12} \mathrm{~A}_{7} \mathrm{H}(49,50)$. Este compuesto cristaliza en el sistema cúbico, con grandes huecos en su estructura cristalina en los cuales pueden alojarse moléculas de $\mathrm{Ca}(\mathrm{OH})_{2}$ de tan pequeño tamaño que no se detectan por DRX por lo que la composición química del conjunto puede representarse por la formula $\mathrm{C}_{3} \mathrm{AH}_{1.5}(52-54)$. Esto podria justificar que no se haya observado la presencia de $\mathrm{Ca}(\mathrm{OH})_{2}$ en el estudio de la deshidratación de cementos refrac-
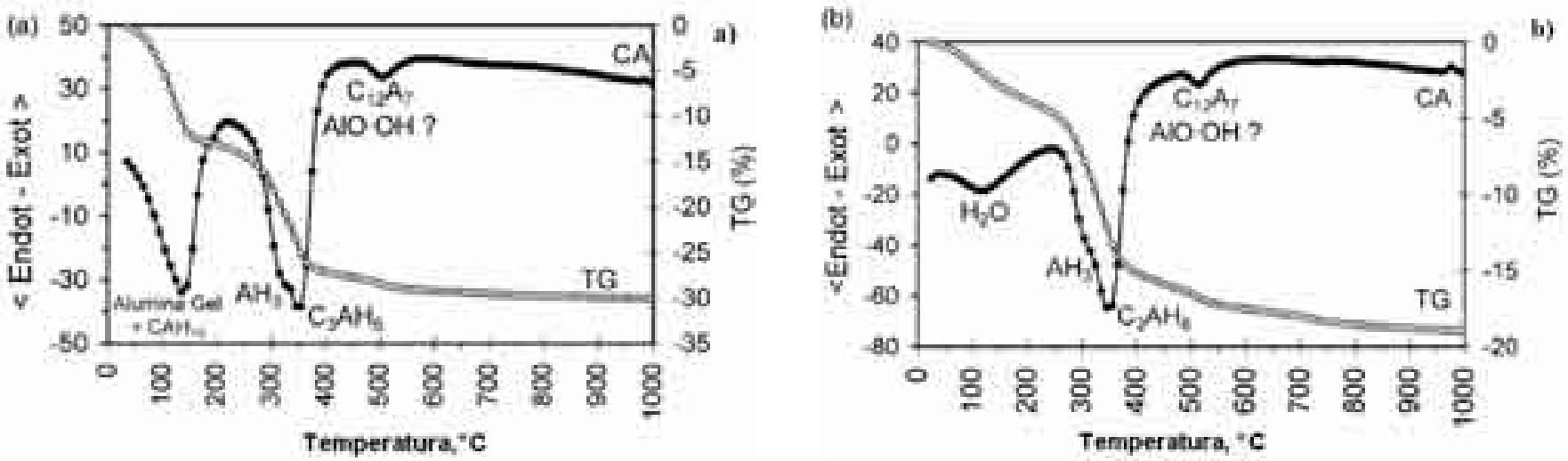

Figura 5-Análisis térmico diferencial y termogravimétrico a $2^{\circ} \mathrm{C} / \mathrm{min}$. $(45,56)$ de:

a) un CA hidratado a $20^{\circ} \mathrm{C}$ que antes del estudio por análisis térmico diferencial estaba compuesto por una mezcla de $\mathrm{CAH}_{10} \mathrm{y}^{\mathrm{A}} \mathrm{H}_{3}$;

b) un CA hidratado y totalmente convertido por lo que antes del estudio por ATD-TG estaba formado por una mezcla de $\mathrm{C}_{3} \mathrm{AH}_{6} \mathrm{y}_{\mathrm{A}} \mathrm{H}_{3}$. 


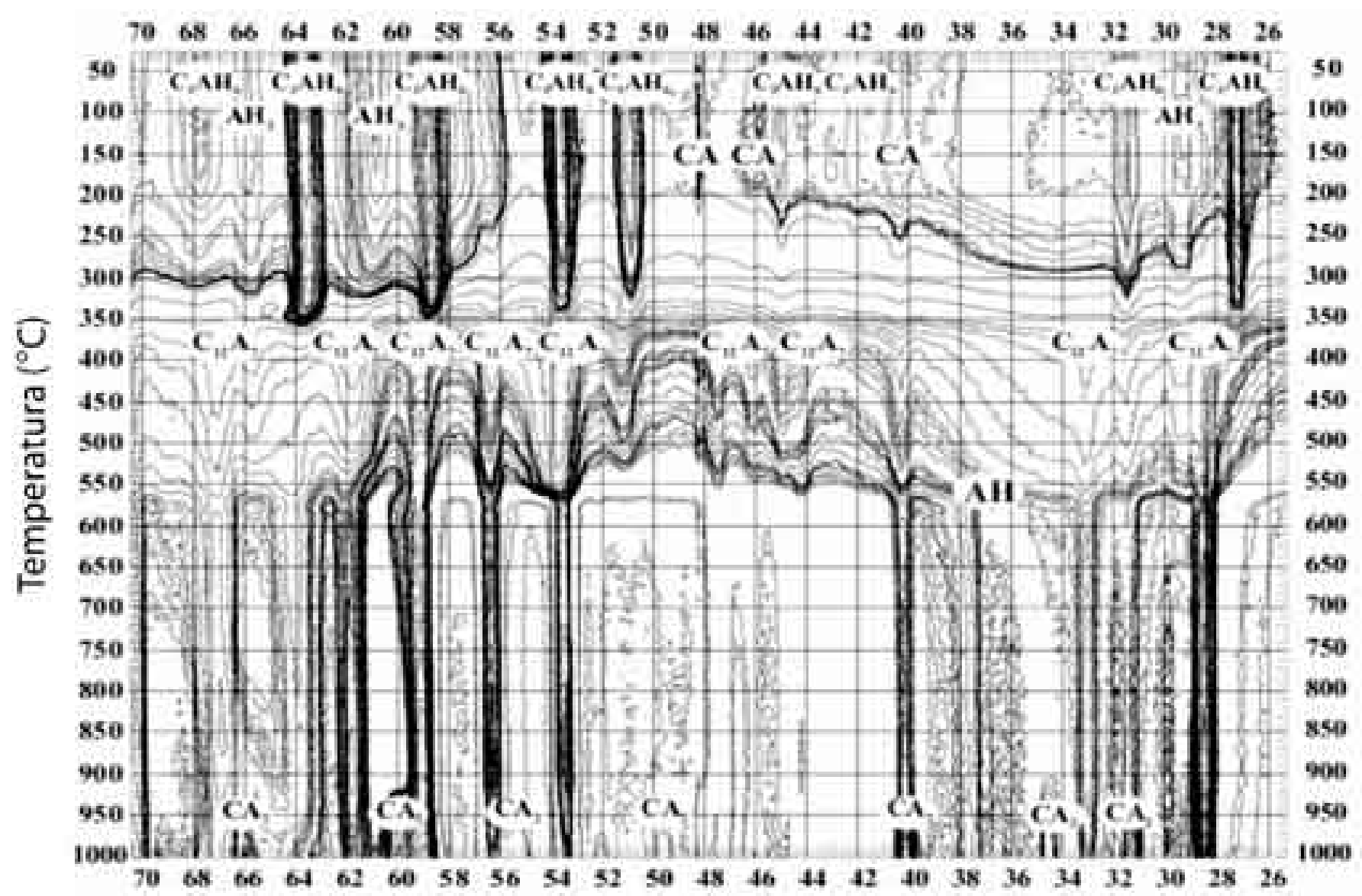

Ángulo $(\Theta)$

Figura 6. Mapa bidimensional proyectado sobre los ejes temperatura ángulo de difracción $(2 \Theta)$ de la secuencia de difractometrias obtenidas durante el calentamiento de una muestra de $\mathrm{CA}$ hidratada con agua deuterada y totalmente convertida en una mezcla de $\mathrm{C}_{3} \mathrm{AH}_{6}$ y $\mathrm{AH}_{3}$. La longitud de onda utilizada en el experimento ha sido $2.4225 \AA$. Se han indicado las fases cristalinas identificadas. El experimento se ha realizado a una velocidad de calentamiento de $2^{\circ} \mathrm{C} / \mathrm{min}$.(45)

tarios de alto contenido en alúmina (45,50). En el intervalo de temperatura entre 360 y $550^{\circ} \mathrm{C}$ el material pierde las restantes moléculas de agua para dar como productos de deshidratación, $\mathrm{C}_{12} \mathrm{~A}_{7} \mathrm{y}$ una alúmina de transición con un muy bajo grado de cristalinidad (Fig. 6) (45). A temperaturas superiores a los $900{ }^{\circ} \mathrm{C}$ la fase $\mathrm{C}_{12} \mathrm{~A}_{7}$ reacciona con las partículas de alúmina altamente reactivas remanentes para formar $\mathrm{CA}_{2}$ comenzando la sinterización y la recuperación de las propiedades mecánicas del material (Fig. 6). El CA $\mathrm{CA}_{2}$ actúa como fase transitoria y a su vez reacciona a $\mathrm{T}>950^{\circ} \mathrm{C}$ con el $\mathrm{C}_{12} \mathrm{~A}_{7}$ para formar $\mathrm{CA}\left(1.080^{\circ}\right.$ C) $(45,54-56)$.

En los sistemas con adiciones de microsilice además de las fases anteriormente descritas, pueden estar presentes $\mathrm{C}_{2} \mathrm{ASH}_{8}$ y soluciones de sólidas de hidrogranates de tipo $\mathrm{C}_{3} \mathrm{~A}(\mathrm{~S})_{3-\mathrm{x}}(\mathrm{H})_{2 \mathrm{x}} \mathrm{o}$ compuestos con estructuras zeoliticas, geles de tipo $\mathrm{CA}(\mathrm{S})_{\mathrm{m}} \mathrm{H}_{\mathrm{n}}$ y sílice sin reaccionar.

No existen en la literatura muchos datos con respecto a la deshidratación de los cementos de aluminatos de calcio con adiciones de microsílice, pero es de suponer que en estos sistemas las fases hidratadas $\mathrm{C}_{3} \mathrm{AH}_{6^{\prime}} \mathrm{C}_{2} \mathrm{AH}_{8^{\prime}} \mathrm{CaAH}_{10}$ y $\mathrm{AH}_{3}$ siguen el mecanismo descrito anteriormente para los cementos de aluminatos de calcio, mientras que la strätlingita (si está presente) pierde el agua unida químicamente alrededor de $210{ }^{\circ} \mathrm{C}$, amorfizandose ${ }^{43}$. Las restantes fases minerales del sistema C-A-S-H presentan una mayor estabilidad dependiendo de su contenido en sílice, liberando agua hasta por encima de los $550{ }^{\circ} \mathrm{C}$ con amorfización posterior. Con el aumento de la temperatura el $\mathrm{Ca}_{12} \mathrm{~A}_{7}$ reacciona con las alúminas de transición y la microsílice disponible para formar $\mathrm{CA}, \mathrm{CA}_{2}, \mathrm{Ca}_{2} \mathrm{AS}$ (gehlenita), $\mathrm{CaAS}_{2}$ (anortita) y $\mathrm{SiO}_{2}$ (cristobalita) a temperaturas alrededor de los $1.000^{\circ} \mathrm{C}$ (57).

Los hormigones refractarios con adiciones de microsílice presentan, a temperaturas entre 1.300 y $1.400{ }^{\circ} \mathrm{C}$ mejores propiedades mecánicas y menor porosidad que los hormigones refractarios sin microsílice. Esta observación se atribuye a la elevada reactividad que presenta la microsílice, y a su capacidad para formar fases liquidas ricas en sílice de elevada viscosidad, que dependiendo de la composición, al alcanzar la saturación pueden originar $\mathrm{C}_{2} \mathrm{AS}, \mathrm{CAS}_{2} \mathrm{o} \mathrm{A}_{3} \mathrm{~S}_{2}$. Si la adición de sílice es idónea se formaran cristales con forma de agujas aciculares interconectadas de mullita, responsables de las mejores propiedades termomecánicas de estos materiales (58). 


\section{CONCLUSIONES}

La adición de alúminas reactivas calcinadas con elevadas superficies específicas, a mezclas de CA y $\mathrm{CA}+\mathrm{CA}_{2}$, acelera la velocidad y el grado de hidratación de los aluminatos de calcio y de sus mezclas, requiriendo especial atención el contenido de $\mathrm{Na}_{2} \mathrm{O}$ presente en su composición.

La microsílice actúa como agente retardador de la hidratación de los aluminatos de calcio, reteniendo iones de $\mathrm{Ca}^{2+}$ $\mathrm{y} \mathrm{Al}^{3+}$, al formar fases minerales dentro del sistema C-A-S-H con estructuras cristalinas que poseen canales abiertos de tipo "pseudo-zeolitas", $\mathrm{C}_{2} \mathrm{ASH}_{8}$ e hidratos de C-S-H.

La hidratación de los cementos de aluminatos de calcio en presencia de sílice coloidal es un proceso complejo, cuyo mecanismo de reacción todavía no esta totalmente comprendido.

La complejidad del diagrama C-A-S-H pone de manifiesto la necesidad de estudios más detallados sobre las relaciones de equilibrio de fases de este sistema que permitan predecir el comportamiento de las fases a baja temperatura y su relación con el proceso de hidratación/deshidratación de los cementos de aluminatos de calcio para aplicaciones refractarias.

Debido a la sensibilidad de las fases que se forman a las condiciones experimentales, la literatura es ambigua tanto con respecto a la formación y estabilidad de la fase $\mathrm{C}_{2} \mathrm{ASH}_{8}$ como también de las soluciones sólidas de la familia de los hidrogranates, lo que pone de nuevo de manifiesto la necesidad de estudios más detallados sobre el sistema C-A-S-H.

\section{AGRADECIMIENTOS}

Este trabajo ha sido subvencionado por la CICYT dentro del proyecto MAT-2000-0941. JMRM agradece al AECI la concesión de una beca.

\section{BIBLIOGRAFÍA}

1. H. Fryda, K. Scrivener, T. Bier, B. Espinosa, «Relation Between Seeting Properties of Low Cement Castables and their Interection with the binder system (CAC-Fillers-admistures-water)», pp. 1315-1323, vol III, en Proceedings of UNITECR 97, Unified International Technical Conference on Refractories, Fifth Biennal Worldwide Congress, New Orleans (EE.UU) (1997).

2. L. Zaigeng, Z. Luming, Y. Guotian y Z. Ningsheng, «The effects of some ultrafine oxides on the properties of ultralow cement corundun-based castables», pp. 149-152, en XXXV, International Colloquium on Refractories, Aachen (Alemania) (1992).

3. N. Bunt, C. Revais, M. Vialle, «Additives in Calcium Aluminates Cement Containing Castables», pp. 1347-1353, vol. III, en Proceedings of UNITCER 97, Unified International Technical Conference on Refractories, Fifth Biennal Worldwide Congress, New Orleans (EE.UU.) (1997).

4. J. M. Rivas Mercury, A. H. De Aza, X. Turrillas y P. Pena, «Hidratación de los cementos de aluminatos de calcio. Parte I». Bol. Soc. Esp. Ceram. V., 42[5], 269-276 (2003).

5. T. J. Carbone, «Production Processes, Properties, and Application for Calcined and High Purity Aluminas», pp. 99-108, en Alumina Chemicals: Science and Technology Handbook, The American Ceramic Society Inc., Westerville, Ohio (EE.UU.), Ed. L. D. Hart, (1990).

6. V. K. Singh, «High-alumina refractory castable with calcium aluminate binder», J. Mater. Sci. Lett, 8 [4] 424-426 (1989).

7. S. I. Odanaka, K. Nakashima, M. Thoj y H. Nagata, «Effect of Alumina Fine Powder and Silica Flour Addition Fluidity of Castable Refractories», Taikabutsu Overseas, 10 [1] (1990).

8. A Rettel, R. Seydel, W. Gessner, J. P. Bayoux y A. Campas, «Investigation on the influence of alumina on the hydration of monocalcium aluminate at differents temperatures», Cem. Concr. Res., 23 [5] 1056-1064 (1993).

9. S. M. Bushnell y J. H. Sharp, «The effect of the temperature upon setting behaviour of refractory calcium aluminate cements», Cem. Concr. Res., 16
[6] 875-884 (1986).

10. S. M. Bushnell y J. H. Sharp, «Further studies of the effect of temperature upon the setting behaviour of refractory calcium aluminate cements», Cem. Conc. Res. 20 [5] 623-635 (1990).

11. S. M. Bushnell y J. H. Sharp, «On the cause of the anomalous setting behaviour with respect to temperature of calcium aluminate cements», Cem. Conc. Res., 20 [5] 677-686 (1990).

12. W. Geßner, S. Möhmel, R. Trettin, A. Rettel and D. Müller, «On the change of microstructure during the hydration of mono calcium aluminate at 20 and $50^{\circ} \mathrm{C}$, pp. 96-109, en Proceedings of the Int. Symp. on Calcium Aluminate Cements, Londres (Inglaterra) 1990.

13. W. Geßner, S. Möhmel, A. Schmalstieg, A. Rettel, J. P. Bayoux, A. Capmas, A. Mathieu, «On the influence of the specific area and $\mathrm{Na}_{2} \mathrm{O}$ content of aluminas on hydration processes in $\mathrm{CaO}{ }^{*} \mathrm{Al}_{2} \mathrm{O}_{3} / \mathrm{Al}_{2} \mathrm{O}_{3}$ mixes», pp. 313-320, en Proceedings of the UNITECR 95, Unified International Technical Conference on Refractories, Fourth Biennal Worldwide Congress, Kioto (Japon) (1995).

14. S. Möhmel, W. Geßner, D. Müller, R. Trettin, G. Oliew, A. Capmas, A. Mathieu, «The hydration of mono calcium aluminate at $50^{\circ} \mathrm{C}$ in presence of aluminas, different in reactivity», pp. 321-328, en Proceedings of the UNITECR 93, Unified International Technical Conference on Refractories, São Paulo, (Brasil) 1993.

15. S. Möhmel, W. Geßner; «The influence of alumina reactivity on the hydration behaviour of mono calcium aluminate», J. Solid State Ionics, (101-103) 937943 (1997).

16. S. Möhmel, W. Geßner, D. Müller, «The influence of aluminas on the behaviour of $\mathrm{CA} / \mathrm{CA}_{2}$ cement during hydration and thermal treatment», pp. 86-89, en Proceedings of the UNITECR 99, Berlin, (Alemania) 1999.

17. A. Mathieu, A. Capmas, J. P. Bayoux y D. Richon, «Calcium Aluminates Cement and Reactive Alumina», pp. 672-682, en Proceedings of the UNITCER 93, Unified International Technical Conference on Refractories, São Paulo, (Brasil) 1993.

18. N. D. Chattherjee, W. Johannes y H. Leistner, «The system $\mathrm{CaO}-\mathrm{Al}_{2} \mathrm{O}_{3}-\mathrm{SiO}_{2}-$ $\mathrm{H}_{2} \mathrm{O}$ : new phase equilibria data, some calculated phase relations, and their petrological applications», Contrib. Mineral Petrol, 88 1-13 (1984).

19. D. Perkins, E. F. Westrum Jr. y E. J. Essene, «The thermodynamic properties and phase relations of some minerals in the system $\mathrm{CaO}-\mathrm{Al}_{2} \mathrm{O}_{3}-\mathrm{SiO}_{2}-\mathrm{H}_{2} \mathrm{O}$ ", Geochimica et Cosmochimica Acta, 44 61-84 (1980).

20. C. C. Addad y P. Ducros, «Étude de la Substition du Agrupament $\mathrm{SiO}_{4}$ par $(\mathrm{OH})_{4}$ dans les Composés $\mathrm{Al}_{2} \mathrm{Ca}_{3}(\mathrm{OH})_{12}$ et $\mathrm{Al}_{2} \mathrm{Ca}_{3}\left(\mathrm{SiO}_{4}\right)_{2.16}(\mathrm{OH})_{3.36}$ de Type Grenat», Acta Cryst., 23 220-230 (1967).

21. G. A. Novak y G. V. Gibbs, «The crystall chemistry of silicate garnets». American Mineralogist, 56 791-825 (1971).

22. D. Damidot y F. P. Glasser, "Investigation of The $\mathrm{CaO}-\mathrm{Al}_{2} \mathrm{O}_{3}-\mathrm{SiO}_{2}-\mathrm{H}_{2} \mathrm{O}$ system at $25^{\circ} \mathrm{C}$ by Thermodynamic Calculations», Cem. Con. Res. 25 [1] 2228 (1995).

23. T. G. Jappy y F. P. Glasser, «Synthesis and Stability of Silica- substituted hydrogarnet $\mathrm{Ca}_{3} \mathrm{Al}_{2} \mathrm{Si}_{3-\mathrm{x}} \mathrm{O}_{12-4 x}(\mathrm{OH})_{4 \times}$ ", Adv. in Cem. Res. 4 [1] 1-8 (1991/92).

24. E. Passaglia y R. Rinaldi, «Katoite, a new member of the $\mathrm{Ca}_{3} \mathrm{Al}_{2}\left(\mathrm{SiO}_{4}\right)_{3}$ $\mathrm{Ca}_{3} \mathrm{Al}_{2}(\mathrm{OH})_{12}$ series and a new nomenclature for thw hydrogrossular group minerals», Bull. Minéral, 107 605-618 (1984).

25. R. C. da Silveira, M. S. Lamas, M. J. A. Cysne, G. Savani, M. A. Minafra, E. F. Godoi y N. Ussem, «Silica Fume CCM - Production and Application», pp. 717-727, en Proceedings of the UNITCER 93, Unified International Technical Conference on Refractories, São Paulo, (Brasil) 1993.

26. A.R. Studart, W. Zhong, R.G. Pileggi, P. Bonadia y V.C Pandolfelli, «Dispersion of Microsilica-Containing Zero-Cement High-Alumina Castables», Am. Ceram. Soc. Bull., 79 [2] 49-55 (2000).

27. B. Moyhre, «Tabular alumina based refractory castables», Parte 3: Reactions in the bond phase of microsilica containing castables», Informe técnico de Elken Refractories, Kristiansand, Noruega.

28. B. Monsen y A. Seltveit, «Effect of Microsilica on Physical Properties and Mineralogical Composition of Refractory Concretes», pp 201-210 vol. 13 en New Developments in Monolithics Refractories (Advances in Ceramics), Ed. R. E Fisher, The American Ceramic Society, Inc., Columbus, Ohio (EE.UU.) 1985.

29. W. Geßner, S. Mohmel, J. Kieser y M. Hawecker, «Investigation of The Composition of Phases Formed in Low Cement Castables During Hydration and After Thermal Treatment», pp. 52-64, en Calcium Aluminates Cements, Ed. R. J. Mangabhai (E. \& FN. SPON), Londres (Inglaterra) 1990.

30. B. Myhre y B. Sunde, «Tabular alumina based refractory castables», Parte 1: The effect of microsilica additions on Flow and cold strech of tabular alumina based low and ultralow cement castables», Informe técnico de Elken Refractories, Kristiansand, Noruega.

31. H. G. Midgley y P. B. Rao, «Formation of Strätlingite, $2 \mathrm{CaO} \cdot \mathrm{SiO}_{2} \cdot \mathrm{Al}_{2} \mathrm{O}_{3} \cdot 8 \mathrm{H}_{2} \mathrm{O}$, in Relation to Hydration of High Alumina Cement», Cem. Conc. Res 8 [2] 169-172 (1978).

32. R. Turriziani, «The Chemistry of Cement», pp. 77, vol. 2, Ed. H. F. H. Taylor, Academic Press, Nueva York (EE.UU). (1974). 
33. S Bentsen, A. Seltveit, and B. Sandberg, «Effect of Microsilica on Conversion of High Alumina Cement», pp. 294-319, en Calcium Aluminates Cements, Ed. R. J. Mangabhai (E. \& FN. SPON), Londres (Inglaterra) 1990.

34. J. Ding, Y. Fu y J. J. Beaudoin, «Strätlingite Formation in High - Alumina Cement - Zeolite Systems», Adv. in Cem. Res. 7 (28) 171-178 (1995).

35. J. Ding, Y. Fu y J. J. Beaudoin, «Strätlingite Formation in High - Alumina Cement - Silica Fume Systems : Significance of Sodium Ions», Cem. Concr. Res. 25 (6) 1311-1319 (1995).

36. J. Ding, Y. Fu y J. J. Beaudoin, «Study of Hydration Mechanism in The High Alumina Cement - Sodium Silicate System», Cem. Concr. Res. 26 (5) 799-804 (1996).

37. Y. Fu, J. Ding, y J. Ding, Y. Fu y J. J. Beaudoin, «Mechanism of Strätlingite Formation in High Alumina Cement - Siliceus Materials Systems», pp. 461471 en Proceeding of Second CANMET/ACI, Int. Conf. On Advances in Concrete Tecnology, Las Vegas (EE.UU.) 1995.

38. D. A. Fumo y A. Segadães, «Effect of Silica Fume Additions on The Hydration Behaviour of Calcium Aluminates», pp. 1325- 1333, vol. III, en Proceedings of The UNITECR 97, Unified International Technical Conference on Refractories, Fifth Biennal Worldwide Congress, New Orleans (EE.UU.) (1997).

39. S. Mohmel, W. Ge $\beta$ ner, J. Kieser y K. Meyer, «Einflu $\beta$ unterschiedlicher Zusätze von $\mathrm{SiO}_{2}$ und $\mathrm{Al}_{2} \mathrm{O}_{3}$ auf die festigkeitsbildenden Reaktionen des Monocalciumaluminats», Keramische Zeitschrift, 47 [9] 692-697 (1995).

40. M. Georgescu y A. Badanoiu, «The Silica Fume and The Temperature Influence on Hydration and The Hardening Process of $12 \mathrm{CaO} .7 \mathrm{Al}_{2} \mathrm{O}_{3} »$, Rev. Roum. de Chemie, 44 [1] 25-30 (1999).

41. S. Mohmel et. al. «The Influence of Microsilica on The Course of Hydration of Monocalcium Aluminate», pp. 319-330, en Proceedings of the International Conference on Calcium Aluminate Cements, Ed. R.J. Mangabhai y F.P. Glasser, Edinburg (Escocia) 2001.

42. D. A. Fumo, «Cimentos em Betoes Refratários LCC e ULCC: Sintése, Mecanismo de Hidrataçao e Interaçao com os Microenchedores», Tesis doctoral, Universidade de Aveiro (1997).

43. W. E. Lee, W. Vieira, S. Zhang, K. Ghanbari, H. Sarpoolaky y C. Parr, «Castable refractory concretes», International Material Review, 46 [3] 145167 (2000).

44. F. Guirado, S. Gali y J. S. Chinchon, «The Thermal Decomposition of Hydrated Alumina Cement $\left(\mathrm{CAH}_{10}\right)$ ", Cem. Concr. Res. 28 [3] 381-390 (1998).
45. X, Turrillas, P. Convert, T. Hansen, A. H. De Aza, P. Pena, «The Dehydration of Calcium Aluminate Hydrates Investigated by Neutron Thermodiffractometry», pp. 517-531, en Proceedings of the International Conference on Calcium Aluminate Cements, Ed. R.J. Mangabhai y F.P. Glasser, Edinburg (Escocia) 2001.

46. Nagai, H; Oshima, K; Hirano, K.; Kato, A., «Sintering Behaviour of Aluminium Hydroxides with Various Morphologies», Brit. Ceram. Trans. 92 [3] 114-118 (1993).

47. Weffers, K.; Bell, G., «Oxides and hidroxides of Aluminum», Technical Paper n. 19, ALCOA - Research Laboratories Center, Pittsburg (EE.UU.) 1987.

48. W. G. Schneider and Thorbergur Thorvaldson, «Dehydration of Tricalcium Aluminate Hexahydrate», Can. J. Res., 19 B 123-128 (1941).

49. W. Bünssem, «X- Rays and Cement Chemistry», pp. 141-168, en Proceedings of The Symposium of Chemical of Cements, Stockholm (Suecia) (1940) .

50. A. J. Majundar y R. Roy, «The system $\mathrm{CaO}-\mathrm{Al}_{2} \mathrm{O}_{3}-\mathrm{H}_{2} \mathrm{O}$ », J. Am. Ceram.Soc. 39 434-442 (1956)

51. M. C. Ball, «The thermal dehydroxylation of $\mathrm{C}_{3} \mathrm{AH}_{6}$ », Cem. Conc. Res. 6 [3] 419-420 (1976)

52. H. J. Kuzel, «Ubre die orientierte Entwässerung von Tricalciumaluminath ydrate $\mathrm{C}_{3} \mathrm{AH}_{6}$ ", Neues Jahrbuch fuer Mineralogie, Monatshefte (9) 397-403 (1969).

53. Bartl, $\mathrm{H}$., «Roentgen-Einkristalluntersuchungen an $(\mathrm{CaO})_{3}\left(\mathrm{Al}_{2} \mathrm{O}_{3}\right)\left(\mathrm{H}_{2} \mathrm{O}\right)_{6}$ und an $(\mathrm{CaO})_{12}\left(\mathrm{Al}_{2} \mathrm{O}_{3}\right)_{7}\left(\mathrm{H}_{2} \mathrm{O}\right)$. Neuer Vorschlag zur $(\mathrm{CaO})_{12}\left(\mathrm{~A}_{12} \mathrm{O}_{3}\right)_{7}$-Struktur», Neues Jahrbuch fuer Mineralogie, Monatshefte (9) 404-413 (1969).

54. N. Richard, «Structure et propriétés élastiques des phases cimentiéres à base de mono-aluminate de calcium», Thesis Doctoral, Universidad de Paris (Francia) 1995.

55. N. Schmitt et al., "Coupling between Kinetics of Dehydration, Physical and Mechanical Behaviour for High Alumina Castable», Cem. Conc. Res. 30 [10] 1597-1607 (2000)

56. A.H. De Aza, P. Pena, M A Rodríguez, R. Torrecillas and S. De Aza., «New Calcium Aluminate Cement containing Magnesium Aluminate Spinel», J. Eur.Ceram. Soc. 23 [1] 737-744 (2003).

57. H. Shikano, J. Yoshitomi, M. Kanda, S. Kititani, M. Sato y Y. Sasagama, «Role of Silica Fluor in Low cement Castable», Taikabutsu Overseas 10 [1] 17- 22 (1990).

58. S. Banerjee, « Castable Refractories», p. 308, Capitulo II, en Monolithic Refractories - A comprehensive Handbook, The American Ceramic Society Inc., Westerville, Ohio (EE.UU.) (1998). 